\title{
Ultra high precision wavefront sensing for Extreme-AO on ELTs
}

\author{
Olivier Guyon ${ }^{1,2, a}$ \\ 1 Subaru Telescope \\ 2 University of Arizona
}

\begin{abstract}
High precision wavefront sensing is the key to detect low mass (potentially rocky) planets with ELTs. Ideally, a wavefront sensor for an Extreme-AO system should be both very sensitive (to allow high speed wavefront correction) and very accurate (to allow precise calibration of residual starlight vs. planet light in the focal plane). I describe two options which meet these requirements: (1) non-linear curvature wavefront sensing is several orders of magnitude more sensitive than conventional WFSs, and can work at full sensitivity in open loop or in the visible (2) focal plane wavefront sensing combines high sensitivity and is free from non-common path errors. It can also measure light coherence, and therefore separate speckles from planets. Combining these two schemes is especially attractive for Extreme-AO systems aimed at direct imaging of exoplanets with ELTs. Laboratory demonstration of both Focal plane wavefront sensing is also be presented.
\end{abstract}

\section{Wavefront sensor sensitivity}

The Wavefront sensor sensitivity is defined as its ability to use a limited number of photon for accurate measurement of wavefront aberrations. In an ideal WFS (optimal sensitivity), for each wavefront mode to be measured, the error in radian RMS on pupil is equal to the inverse square root of the number of photon. This simple equation can be generalized to a wavefront measurements of M modes with a total of $\mathrm{N}$ photon:

$$
\sigma(r a d)=\sqrt{M / N}
$$

Direct comparison between this ideal limit and current wavefront sensing schemes reveals a huge performance gap, especially at low spatial frequencies. Commonly used wavefront sensors such as Shack-Hartmann and Curvature are very robust and flexible, but are poorly suited to high sensitivity wavefront measurement: for low-order aberrations which prevent high contrast imaging very close to a star they require $\approx((p / 2) / r 0)^{2}$ (with $\mathrm{p}=$ spatial period of the aberration) more photons than the theoretical limit above. This is a factor 100 to 1000 on 8 to $10-\mathrm{m}$ telescopes. These conventional wavefront sensing schemes offer nearly ideal sensitivity at a single spatial frequency (defined by the WFS subaperture spacing for SH and curvature) but suffer from poor sensitivity at low spatial frequencies (an effect commonly referred to as "noise propagation"), which are most critical for direct high contrast imaging of exoplanets and disks.

WFS schemes offering very high sensitivity across a wide range of spatial frequencies have previously been proposed: Mach-Zehnder pupil plane interferometry (Angel 1994), Zernike phase contrast wavefront sensing (Zernike 1934, Bloemhof \& Wallace 2003), pyramid wavefront sensing (Ragazzoni 1996). These WFSs concepts do indeed offer sensitivity at or very close to the theoretical ideal limit across a wide range of spatial frequencies (Guyon 2005), but only in the small aberration regime where the wavefront error at the sensing wavelength is below $\approx 1 \mathrm{rad}$ RMS ( $=87 \mathrm{~nm} \mathrm{RMS} \mathrm{in} \mathrm{V} \mathrm{band)} \mathrm{at} \mathrm{the}$ sensing wavelength.

In $\S 2$, I describe a new type of wavefront sensor which, for the first time, offers full sensitivity without requiring the wavefront to be nearly flat at the sensing wavelength. The approach is derived from the already highly successful curvature wavefront sensing technique, but uses a non-linear wavefront reconstruction algorithm (non-linearity is unavoidable for simultaneous delivery of high sensitivity

\footnotetext{
a e-mail: guyon@naoj .org
}

This is an Open Access article distributed under the terms of the Creative Commons Attribution-Noncommercial License, which permits unrestricted use, distribution, and reproduction in any noncommercial medium, provided the original work is properly cited. 


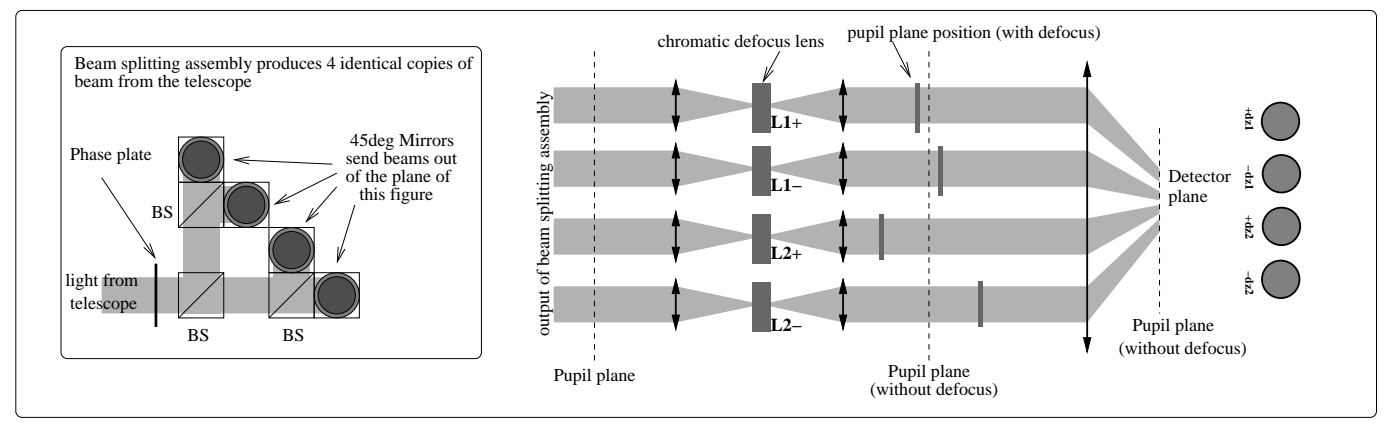

Fig. 1. Proposed optical layout for non-linear curvature wavefront sensing measurement.

and wide dynamic range). In $\S 3$, I discuss wavefront sesing from focal-plane images using an upstream Deformable Mirror. I show recent laboratory results demonstrating both high contrast imaging and accurate calibration of residual light with this technique.

\section{Non-linear Curvature Wavefront Sensing}

\subsection{Principle}

Figure 1 shows a possible optical implementation for the non-linear Curvature WFS (nlCWFS). The left panel shows collimated light entering from the bottom left, and going though a series of four beam splitters immediately followed by four 45-deg mirrors. This assembly produces four identical copies of the telescope beam. The right part of the figure shows these four beam going through a set of optics designed to re-image the four pupil planes on a common detector. The four pupil images can be independently defocused by adding a powered element in an intermediate focal plane (this is the static equivalent of a vibrating reflective membrane in the focal plane of a conventional curvature WFS).

The four main departures from a conventional curvature WFS are:

- Images are acquired in planes which are considerably further away from the pupil plane, where non-linear effects are strong. This is essential for efficient conversion of phase into intensity signal at all spatial frequencies when a limited number of photon is available.

- While curvature systems use a variable focal length vibrating membrane, here, beam splitters and lenses are used to produce the required conjugation planes on the detector(s) array(s). The speckles in the defocused images are then kept very sharp, while a vibrating membrane scheme would have smeared them due to the constant motion of the re-imaged plane in conjugation space. This scheme also allows faster data acquisition: in the figure, the four pupils are lined up and could be placed at the bottom of the detector array to minimize the number of lines to be read.

- Chromatic lenses are used in the sensor's focal planes for compensation of chromatic effects. Fresnel propagation is chromatic, but can be to the first order exactly compensated if the propagation distance from the pupil plane is proportional to wavelength. Figure 5 below illustrates the benefit of this chromatic compensation, which allows broadband (more than 50\%) operation with negligible loss in performance. Chromatic compensation is especially good at preserving small $\lambda / \mathrm{D}$ speckles in images conjugated far away (several thousand $\mathrm{km}$ ) from the pupil plane.

- Four conjugation planes are created instead of two in a conventional curvature WFS. With only two symmetric planes (conjugations $+d z 1$ and $-d z 1$ ), there is a spatial frequency for which the propagation distance is equal to the Talbot distance: for this frequency, phase aberrations are not converted into intensity signals. This problem is solved with two different propagation distances (four images total, at conjugations $+\mathrm{dz} 1,-\mathrm{dz} 1,+\mathrm{dz} 2$ and $-\mathrm{dz} 2$ ). We confirmed by numerical simulations that closed loop performance was significantly lower if the number of planes is reduced below 4 . 


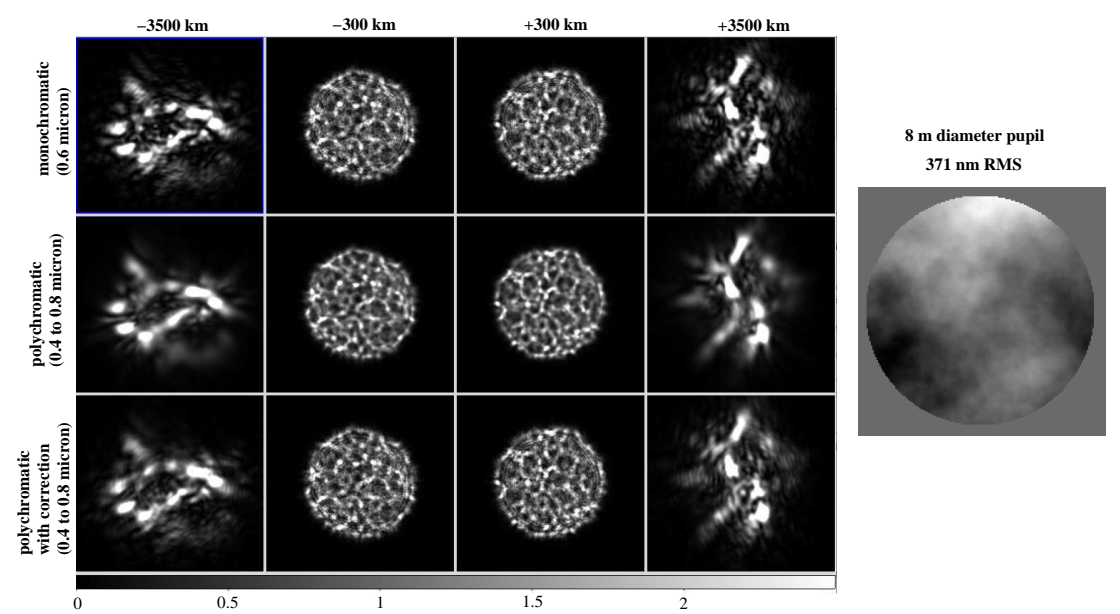

Fig. 2. Simulated nlCWFS data. Top: monochromatic light. Center: in polychromatic light, speckles are blurred. Bottom: with chromatic compensation built in the optical design, speckles are sharp in polychromatic light.

Figure 2 shows simulated signal obtained with the WFS fot a $371 \mathrm{~nm}$ RMS wavefront error. Each of the 4 images obtained is speckles, and the non-linear Curvature WFS achieves its sensitivity by tracking these diffraction limited speckles. For example, tip-tilt measurement sensitivity is driven by the size of these speckles $(\lambda / D)$ rather than the seeing-limited size $\lambda / r_{0}$ for conventional WFSs. The corresponding gain in flux is equal to $\left(D / r_{0}\right)^{2}$. Figure 2 also shows that chromatic compensation in the optical design is essential to preserve the WFS's sensitivity in broadband light.

The wavefront may be reconstructed from several defocused pupil images through a phase diversity algorithm (Gonsalves \& Chidlaw 1979). For the preliminary work shown in this paper, I have chosen to use a Gerchberg-Saxton [1] algorithm for coding simplicity and flexibility, but many other options and variations on this algorithm are available, as much work has been devoted to phase retrieval algorithms in the last few decades.

Phase unwrapping is also necessary when wavefront errors excede one wavelength. A linear phase unwrapping algorithm decomposing the wavefront slope on a precomputed set of mode was chosen for execution speed.

\subsection{Closed loop simulations}

Figure 3 compares closed loop AO performance obtained with SHWFS and nlCWFS for a total flux of $3 \mathrm{E} 6 \mathrm{ph} / \mathrm{s}$ on a 8 -m telescope (magnitude $\approx 13$ with $20 \%$ efficiency and a $0.5 \mu \mathrm{m}$ wide bandpass). This simulation was performed with a 0.6 arcsecond seeing at $0.5 \mu \mathrm{m}$ and a $10 \mathrm{~m} / \mathrm{s}$ wind speed. Both the WFS and imaging wavelength are $0.85 \mu \mathrm{m}$. The WFS detector array is considered perfect (no readout noise, no dark current). For the SH systems, each subaperture's PSF is imaged on $64 \times 64$ pixels, and the DM is considered ideal, correcting all spatial frequencies measured. Four SHWFS configurations were simulated with $\mathrm{D} / \mathrm{d}$ varying from 60 to $9(\mathrm{D}=$ telescope diameter, $\mathrm{d}=$ subaperture size $)$. For the nlCWFS simulation, the detector acquires 4 images with 90 pixels across the pupil each, and the DM perfectly corrects modes up to 16 cycles per aperture (CPA).

In each case, AO loop frequency was optimized to minimize residual wavefront aberration. Figure 3 illustrates the effect of noise propagation in a SHWFS: with a large number of subapertures, performance on low order aberrations becomes poor, and overall wavefront RMS error becomes large even though the number of modes corrected is high. For a given guide star brightness, there is an optimal number (higher number for brighter stars) of subapertures in a SHWFS for which sensitivity loss on low order aberrations is balanced against number of modes measured. With the nlCWFS, increasing the number of wavefront modes measured does not lower performance for low order aberrations. Correction is very good over the full range of spatial frequencies measured. 


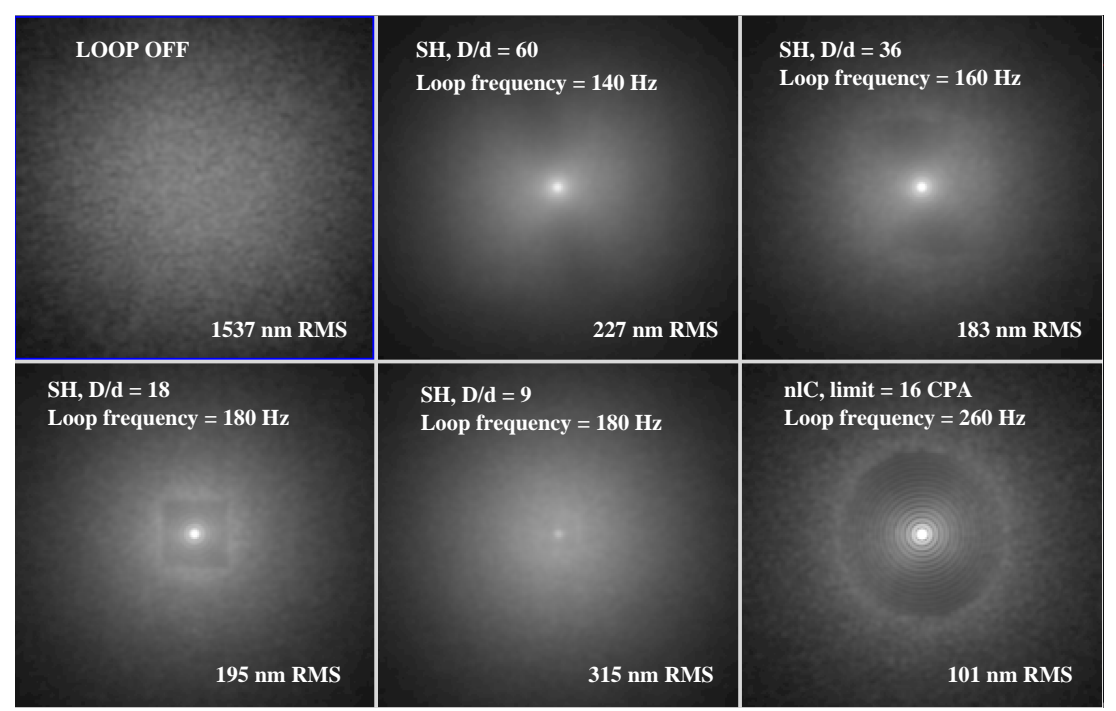

Fig. 3. Closed loop simulations comparing the non-linear Curvature WFS (bottom right) to SH type WFS with several choices of subaperture size $d$.

Table 1. Closed loop simulation results.

\begin{tabular}{lcccc}
\hline & Loop frequ & RMS & SR at $0.85 \mu \mathrm{m}$ & SR at $1.6 \mu \mathrm{m}$ \\
\hline nlCurv & $260 \mathrm{~Hz}$ & $101 \mathrm{~nm}$ & $57 \%$ & $85 \%$ \\
SH - D/d $=9$ & $180 \mathrm{~Hz}$ & $315 \mathrm{~nm}$ & $\approx 4 \%$ & $22 \%$ \\
SH - D/d = 18 & $180 \mathrm{~Hz}$ & $195 \mathrm{~nm}$ & $\approx 13 \%$ & $56 \%$ \\
SH - D/d = 36 & $160 \mathrm{~Hz}$ & $183 \mathrm{~nm}$ & $\approx 16 \%$ & $60 \%$ \\
SH - D/d $=60$ & $140 \mathrm{~Hz}$ & $227 \mathrm{~nm}$ & $\approx 6 \%$ & $45 \%$ \\
\hline
\end{tabular}

Closed loop simulations using the nlCWFS show a rapid drop in performance at about $m_{V} \approx 14$. This limit is due to a loss of coherence in the pupil plane: the number of photons becomes too low to track the diffraction-limited speckles which are essential to the nlCWFS's sensitivity. Beyond this limit, the nlCWFS performance is similar to conventional linear WFSs. Simulations also show that moving the sensing wavelength to the near-IR improves the closed-loop performance (at fixed photon arrival rate) thanks to the recovery of coherence in the pupil plane. A near-IR version of the nICWFS would therefore offer a fainter guide star limit and better sky coverage.

\section{3 nICWFS for Extreme-AO}

The nlCWFS is ideally suited for high contrast coronagraphic imaging. For this application, exquisite control of low order aberrations is most critical, and can enable exoplanet imaging at small angular separations with high performance coronagraphs which, up to now, have only worked well in laboratory experiments. Computation speed for nlCWFS wavefront reconstruction becomes less challenging as wavefront quality improves. With less than $1 \mathrm{rad}$ RMS wavefront aberration (a realistic assumption for a high performance Extreme-AO system), the nlCWFS signal becomes a linear function of phase aberrations, allowing for a simpler and faster reconstructor. Initial locking of the AO loop to approach this linear regime may be an issue, and will be studied as part of our proposed effort.

The raw contrast delivered by an optimally tuned closed loop Extreme-AO system is proportional to $F^{-2 / 3}$, where $\mathrm{F}$ is the guide star flux (equation 20 in Guyon 2005). For an aberration at 2 cycles per aperture (CPA) on an 8-m telescope, the nlCWFS achieves the same accuracy as a D/d=16 SHWFS 
using $\approx 500$ times more photons (assuming spot size in the SHWFS is consistent with 20 rad RMS wavefront aberration): the nlCWFS can reach a contrast ratio 62 times better than a SHWFS, assuming no other limitation. On larger telescopes, the performance difference is even wider, due to the larger difference between the seeing size which defines the SHWFS sensitivity and the telescope diffraction limit which defines the nlCWFS sensitivity.

\section{Focal plane Wavefront Sensing for coronagraphy}

\subsection{Principle}

In Focal plane Wavefront Sensing (fpWFS), wavefront aberrations in a coronagraphic system are inferred from the speckles left in the focal plane. The goal of the technique is to create a dark region in the focal plane (preferably very close to the star) where speckles are iteratively destroyed by moving coherent anti-speckles (speckles with opposite complex amplitudes) on top of existing speckles.

Coherent scattered light in the focal plane mask is measured by phase diversity introduced on the DM. A series of focal plane images, each acquired with a slightly different DM shape, is used to reconstruct the complex amplitude and coherence of the scattered light. A linearized representation of the system in focal plane complex amplitude can be used, as described in the electric field conjugation (EFC) approach proposed by [2]. The wavefront control loop shown in Figure 4 is built around the EFC approach.

Prior to starting the loop, a model of the coronagraph is used to compute how each actuator motion affects the complex amplitude in the focal plane. Since this relationship is linear for small displacements, this model is stored as a complex amplitude system response matrix $M_{\text {resp }}$ (shown on the right hand side of Figure 4) of size $n$ by $m$, where $n$ is the number of DM actuators (ignoring actuators outside the pupil) and $m$ is the number of pixels in the high contrast region of the focal plane. $M_{\text {resp }}$ is the linear operator which establishes the relationship between deformable mirror actuator displacements $\delta D M(u, v)$ (sampled in $n$ points) and the corresponding complex amplitude change $\delta f(x, y)$ (sampled in $m$ points) in the focal plane:

$$
\delta f(x, y)=M_{\text {resp }} \delta D M(u, v) .
$$

$M_{\text {resp }}$ is therefore computed by moving each actuator of the DM in the coronagraph model and storing the corresponding change in focal plane complex amplitude in a column of $M_{\text {resp }}$.

A powerful extension of the EFC algorithm described above is the ability to continuously measure coherent and incoherent light in the focal plane. This is especially attractive as a scheme to separate planet light (incoherent) from speckles (coherent). The coherent light which varies from exposure to exposure can also be measured from the residual left after fitting the WFS data with a model including coherent and incoherent light. The algorithm shown in Figure 4 also shows, on the right, a "self calibration" loop which constantly optimizes $M_{\text {resp }}$ to best fit the data acquired.

\subsection{Laboratory results}

Figure 5 shows results obtained in the laboratory with a PIAA type coronagraph [3] and the wavefront/calibration algorithm described in the previous section. The following main components to the images were measured :

- Incoherent light component: The raw contrast is dominated by a very stable incoherent component at $1.6310^{-7}$, which is likely due to ghosts and/or polarization missmatch. The data obtained is compatible with a fully static incoherent background, as would be expected from ghosts and polarization effects. We note that a fast varying coherent component could also be responsible for this light if it is varying sufficiently rapidly to appear incoherent within $\approx 1$ second.

- Coherent light component: During the measurement sequence, coherent light varies by $\approx 4.510^{-8}$ in contrast due to turbulence or vibrations in the system, as shown by the $\chi^{2}$ analysis. The coherent light leak estimate over a 10 second period is $\approx 510^{-8}$, which is at the level expected from the 4.5 $10^{-8}$ variations shown by the $\chi^{2}$ analysis. The large variation, from iteration to iteration, observed in the coherent light residual is due to the turbulence/vibrations in the system. 


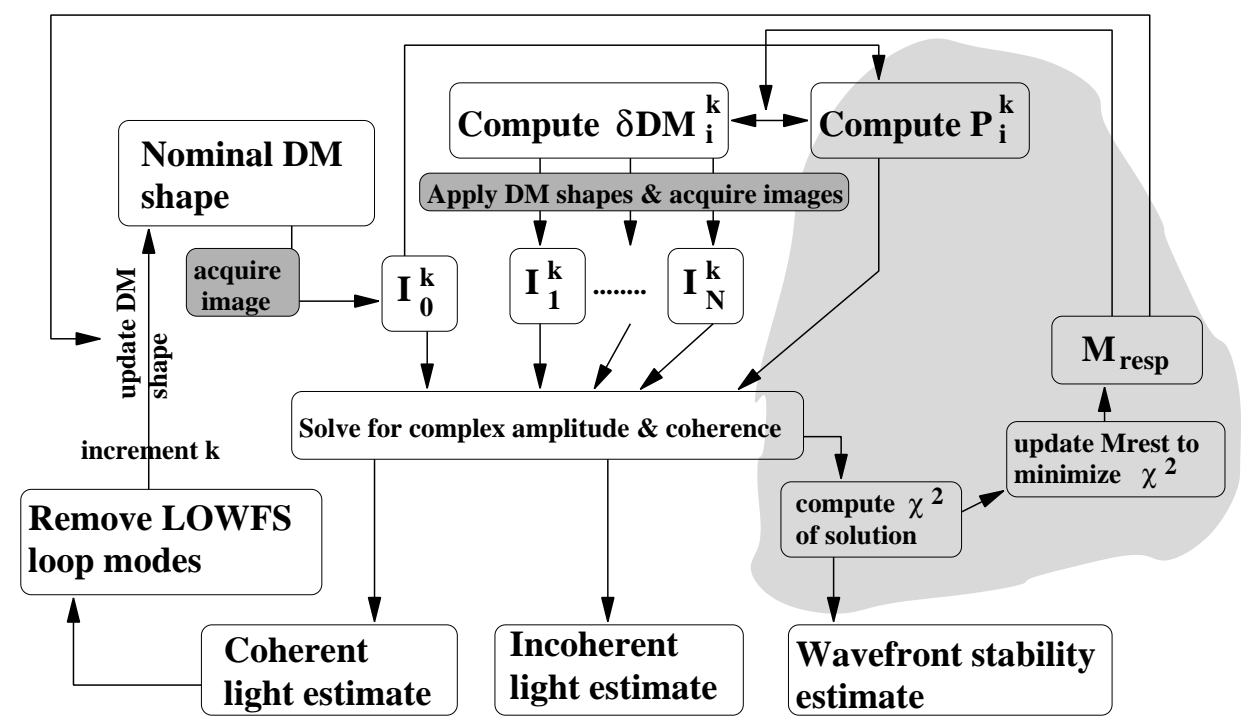

Fig. 4. Focal plane wavefront control algorithm described in this paper. This algorithm simultaneously estimates coherent light (to be corrected by the deformable mirror) and incoherent light.
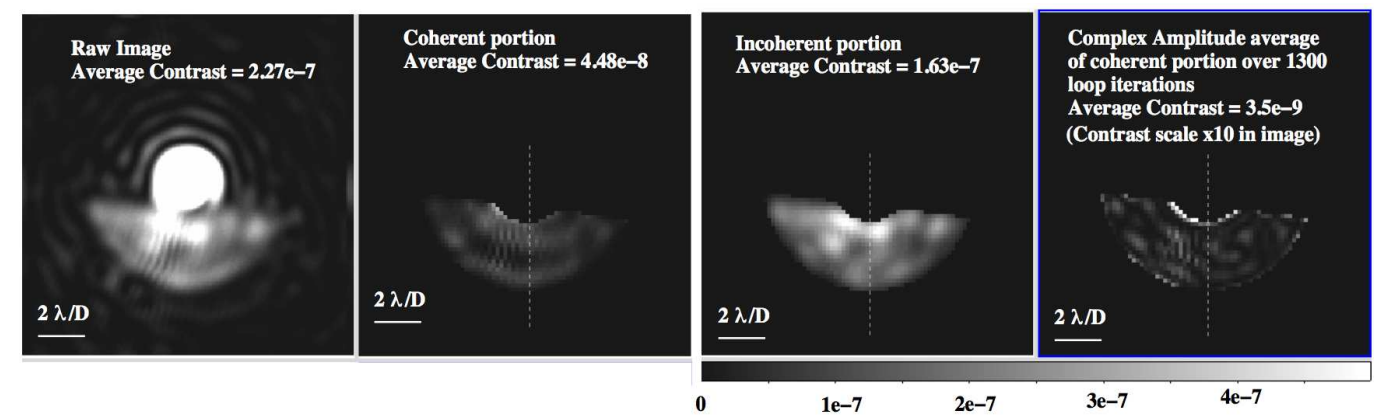

Fig. 5. Left: Raw coronagraphic image. A decomposition of the scattered light into a coherent component (center left) and incoherent component (center right) shows that the raw contrast is dominated by incoherent light. The coherent "bias" over a long period of time is shown in the right.

- Static wavefront aberrations: The wavefront control loop successfully removes static coherent speckles. Over a $4 \mathrm{hr}$ period of time, we have measured the static coherent speckles to be at the $3.510^{-9}$ contrast level. Except for a known ghost on the camera window, we could not detect any residual bias above this level in the residual time-averaged coherent light.

\section{References}

1. Gerchberg, R.W., Saxton, W.O., Optik, 35, 1972

2. Give'on, A. et al., Proc. SPIE, 6691, 2007

3. Guyon, O. , A\&A, 404, 2003 